Methods A survey was designed and sent by email to neonatal units which were applying nCPAP. The survey collected information about the devices, the indications of use and the ventilatory parameters used when delivering nasal ventilation over 2010. It was also questioned whether a guideline was followed.

Results 87 out of 115 questionnaires were answered and returned $(75.6 \%)$. All the surveyed units used nCPAP and the most frequent indications were: apnoea treatment $(87 / 87 ; 100 \%)$ and respiratory distress before surfactant therapy $(85 / 8 ; 97.7 \%) .71$ units used nIPPV $(81.6 \%)$ in order to succeed in extubation $(66 / 71 ; 92.9 \%)$ and for treatment of apnoeas $(63 / 71 ; 88.7 \%)$. Most of the units used variable flow devices to deliver nCPAP (64/87; 73.5\%) and nIPPV (48/71; 67.6\%). 72 units $(82.7 \%)$ followed national guidelines at the time of starting non invasive ventilation. The most used interface was short binasal prongs (58/87; 66.6\%).

Conclusion Both nCPAP and nIPPV are significantly used in Spanish neonatal units following the recommendations of the available national guidelines.

\section{RANDOMISED TRIAL OF SINGLE NASAL PRONG OR FACE MASK FOR RESPIRATORY SUPPORT FOR PRETERM INFANTS IN DELIVERY ROOM (ISRCTN59061709)}

doi:10.1136/archdischild-2012-302724.1802

1.2.3 LK McCarthy, ${ }^{1,2 E J}$ Molloy, ${ }^{1} \mathrm{AR}$ Twomey, ${ }^{1} \mathrm{JF}$ Murphy, ${ }^{1,2,3 \mathrm{CP}} \mathrm{O}^{\prime}$ Donnell. ${ }^{1}$ Department of Neonatology, The National Maternity Hospital; ${ }^{2}$ The National Children's Research Centre, Crumlin; 'University College Dublin, Dublin, Ireland

Background ILCOR recommends that newborns with inadequate breathing or $\mathrm{HR}<100 \mathrm{bpm}$ be given respiratory support via a face mask in the delivery room (DR); however, it may be more effective if given to preterm infants via a single nasal prong (AKA short nasal tube, nasopharyngeal tube).

Aims To determine whether giving respiratory support to preterm infants via a nasal prong rather than a face mask results in fewer infants being intubated in the DR.

Methods Normally formed infants $<31$ weeks' are eligible for inclusion. Randomisation is stratified by gestational age $(<28$ weeks, $\left.28-30^{+6}\right)$ and allocation is concealed in sealed opaque envelopes. With parental consent, infants are randomised just prior to delivery to single nasal prong (ETT shortened to $5 \mathrm{~cm}$ ) or face mask (Fisher \& Paykel, Auckland NZ). Infants who have apnoea, respiratory distress and/or a HR $<100 \mathrm{bpm}$ receive respiratory support with a t-piece. Infants are only intubated in the DR for apnoea and/ or bradycardia despite PPV, not for surfactant administration. All other aspects of treatment in the DR and NICU are the same for both groups. Relevant secondary outcomes are recorded.

Results Since enrollment began (19.07.2010), 121 infants have been recruited and had the primary outcome determined. We expect the primary outcome will be determinable for the total sample of 142 infants by August 2012.

Conclusions This randomised trial will provide valuable information about the preferred interface to use when giving respiratory support to newborn preterm infants in the DR.

\section{DOES VOLUME OF THE MASK VARY AND INFLUENCE MEASUREMENTS DURING NEONATAL RESUSCITATION?}

doi:10.1136/archdischild-2012-302724.1803

${ }^{1} \mathrm{JJ}$ van Vonderen, ${ }^{1} \mathrm{R}$ Kamar, ${ }^{1} \mathrm{~K}$ Schilleman, ${ }^{2} \mathrm{SB}$ Hooper, ${ }^{1} \mathrm{FJ}$ Walther, ${ }^{1} \mathrm{AB}$ te Pas. 'Division of Neonatology, Department of Pediatrics, Leiden University Medical Center, Leiden, The Netherlands; ${ }^{2}$ Ritchie Center, Monash University, Melbourne, VIC, Australia

Background Respiratory function monitoring (RFM) could improve the efficiency of mask ventilation in preterm infants at birth. However, dead space of a mask could vary, depending on rate of pressurization and variation in hand hold, influencing measurements.

Aim To investigate whether mask volume varies during mask ventilation and influences measured tidal volumes and calculated mask leak.

Methods Thirty caregivers of the neonatal unit were asked to mask-ventilate a leak free manikin with pressures $25 / 5 \mathrm{~cm} \mathrm{H}_{2} \mathrm{O}$ and a gas flow rate of 6 and $10 \mathrm{~L} / \mathrm{min}$. A Laerdal 0/1 mask $(40 \mathrm{~mL})$ was glued leak free on the face in the right position but the participant was unaware why the mask position was fixed. The participant was told that mask hold, not positioning, was tested and that it was still possible to have leak. Tidal volumes were measured using a RFM.

Results Inspired tidal volume $\left(\mathrm{V}_{\mathrm{T}_{\mathrm{i}}}\right)$ increased from $8.05 \mathrm{~mL}(0.76)$ at $6 \mathrm{~L} / \mathrm{min}$ to $8.76 \mathrm{~mL}(0.75)$ at $10 \mathrm{~L} / \mathrm{min}(\mathrm{p}<0.01)$ and expired tidal volume $\left(\mathrm{V}_{\mathrm{Te}}\right)$ from $\left.8.15 \mathrm{~mL}(0.81)\right)$ at $6 \mathrm{~L} / \mathrm{min}$ to $8.85 \mathrm{~mL}(0.75)$ at 10 $\mathrm{L} / \mathrm{min}(\mathrm{p}<0.001)$. Median (IOR) leak was $-0.90(-3.90-1.40) \%$ with $6 \mathrm{~L} / \mathrm{min}$ and did not increase with $10 \mathrm{~L} / \mathrm{min}(-0.62(-3.43-1.80) \%$; $\mathrm{ns})$ Coefficient of variance showed good to acceptable agreement for all results.

Conclusion During mask ventilation there is very little variation in mask volume which does not influence respiratory function monitoring.

\section{WHICH MUSICAL TUNE IMPROVES SYNCHRONIZATION OF RESPIRATORY SUPPORT DURING SIMULATED CARDIO- PULMONARY RESUSCITATION OF NEONATES?}

doi:10.1136/archdischild-2012-302724.1804

${ }^{1,2,3} \mathrm{G}$ Schmölzer, ${ }^{4,5} \mathrm{CC}$ Roehr, ${ }^{2} \mathrm{C}$ Wong, ${ }^{2,6} \mathrm{~J}$ Dawson, ${ }^{2,6} \mathrm{P}$ Davis. 'Department of Pediatrics, University of Alberta, Edmonton, AB, Canada; ${ }^{2}$ Neonatal Services, The Royal Women's Hospital, Melbourne, VIC, Australia; ${ }^{3}$ Department of Paediatrics, Medical University of Graz, Graz, Austria; ${ }^{4}$ Department of Neonatology; ${ }^{5}$ Dieter-Scheffner Center for Evidence-Based Medical Education, Charité University Medical Centre, Berlin, Germany; ${ }^{6}$ Department. of Obstetrics \& Gynaecology, The University of Melbourne Melbourne, VIC, Australia

Introduction The need to provide chest compressions and assisted inflations occurs infrequently during neonatal resuscitation. A mannequin study of cardiopulmonary resuscitation (CPR) in adults showed that listening to music improved the coordination of inflations and chest compressions.

Aim To compare several musical tunes during simulated CPR and the effect on coordinating inflations and chest compression during two helper CPR.

Methods Five different tunes ("I will survive" (120 bpm), "Radetzkimarsch" (105 bpm), "Jingle Bells" (120 bpm), "Stayin' alive" (105 bpm), and "S.O.S." (120 bpm)) were played during simulated neonatal CPR. The order in which the tunes were played was randomized. Mask leak and tidal volume was measured using a respiratory function monitor and used to investigate the degree of synchronization of two-helper CPR. Measurements were recorded at baseline (no music) and with individual tunes, each played for one minute during which CPR was provided by neonatal staff.

Results During baseline median (SD) chest compressions and inflations were 80 (6) and 28 (2) per minute, respectively. 43\% of chest compressions occurred during expiration, $16 \%$ during inspiration and $41 \%$ between expiration and inspiration. Only listening to "S.O.S." improved the number of delivered chest compressions and inflations significantly compared to baseline. Mask leak and tidal volume delivery was similar while listening to any of the five musical tunes.

Conclusion ABBA's S.O.S significantly improved the number of chest compressions and inflations. Musical mnemonics apparently have the potential to improve mask ventilation when cardiac compressions are required. Their use should be further investigated. 
1805 OXYGEN SATURATION MONITORING AT BIRTH: FEASIBILITY OF THE 2010 NEONATAL RESUSCITATION GUIDELINES

doi:10.1136/archdischild-2012-302724.1805

'V Dal Cengio, ${ }^{2} \mathrm{M}$ Parotto, 'P Zanella, 'N Rizzo, ' $\mathrm{C}$ Zacchettin, ${ }^{3} \mathrm{~F}$ Cavallin, ${ }^{1} \mathrm{D}$ Trevisanuto, ${ }^{4} \mathrm{~V}$ Zanardo. 'Children and Women's Health Department; 'Department of Anesthesia and Pharmacological Sciences, Padua University School of Medicine; ${ }^{3}$ Independent Statistician, Padova; ${ }^{4}$ Service of Neonatology, Abano Terme General Hospital, Abano Terme, Italy

Background The 2010 Neonatal Resuscitation Guidelines recommend preductal transcutaneous oxygen saturation $\left(\mathrm{SpO}_{2}\right)$ monitoring at birth.

Objective To verify the feasibility of $\mathrm{SpO}_{2}$ monitoring at birth by determining the time to get the first $\mathrm{SpO}_{2}$ value using a pulse oximeter.

Methods The study included 100 healthy newborns at term by elective caesarean section (Elective CS, 50 neonates), vaginal delivery (VD, 32 neonates) and emergency caesarean section (Emergency CS, 18 neonates). A Masimo Radical-7 (Masimo, Irvine, CA) pulse oximeter sensor was applied on neonatal right hand noting the minute at which the first oximetry value was provided. For the comparison between the time to get the first oximetry value among the three groups, Chi Square and Fisher Exact Test were used. A p value $<0.05$ was considered statistically significant.

Results In the total study population, $52 \%$ of $\mathrm{SpO}_{2}$ values were obtained within the first minute of life; $28 \%$ in the second; $13 \%$ in the third; $3 \%$ in the forth; $3 \%$ in the fifth; $1 \%$ in the sixth.

However, the first $\mathrm{SpO}_{2}$ value was more frequently obtained within the first minute of life in newborns by Elective CS (74\%) and by Emergency CS (61\%) than in those by VD (12.5\%), $\mathrm{p}<0.05$.

Conclusions The first minute after birth is critical for Apgar score and neonatal resuscitation. This study demonstrated that $\mathrm{SpO}_{2}$ is not always rapidly measurable, especially in neonates born by VD. A change in current clinical practice is therefore required.

\section{MOLECULAR MECHANISMS OF PERINATAL LUNG FLUID CLEARANCE IN TERM NEWBORNS}

doi:10.1136/archdischild-2012-302724.1806

C Janér, 0 Helve, L Süvari, OM Pitkänen, S Andersson. Children's Hospital, Helsinki University Central Hospital, Helsinki, Finland

Background and Aim The perinatal switch from secretion to absorption in airway fluid transport includes increase in gene expression and activity of ion channels, e.g. apical amiloride-sensitive epithelial sodium channel (ENaC) and basolateral $\mathrm{Na}-\mathrm{K}-\mathrm{ATPase}$. The serum- and glucocorticoid-induced kinase (SGK) may induce $\mathrm{ENaC}$ and $\mathrm{Na}$-K-ATPase.

Our objective was to study airway expression of SGK1, Na-KATPase $\alpha 1$-subunit and $\alpha \mathrm{ENaC}$ during adaptation in term infants.

Methods 86 term infants $(G A=39.43 \pm 0.91$; mean $\pm S D)$ were included in the study (vaginal delivery, $\mathrm{VD}, \mathrm{n}=25$ and elective cesarean section, CS, $\mathrm{n}=61$ ). Within 3 hours and at 22-29 hours after delivery airway cell samples were obtained from the infants' nasal epithelium. $\alpha \mathrm{ENaC}, \mathrm{Na}-\mathrm{K}-\mathrm{ATPase} \alpha 1$-subunit, and SGK1 mRNAs in the samples were quantified with real-time RT-PCR and normalized to cytokeratin 18 (CK18).

Results $\mathrm{ENaC}$ and $\mathrm{Na}-\mathrm{K}$-ATPase $\alpha$-subunit mRNA amounts were similar after VD and CS. During the first postnatal day Na-K-ATPase $\alpha 1$ gene expression decreased in infants delivered by CS $(p<0.001)$. After CS SGK1 mRNA was higher at $<30$ min than at $1-3$ hours of age $(p<0.001)$. Within 3 hours after vaginal delivery $\mathrm{ENaC}$ and
Na-K-ATPase $\alpha$-subunit mRNA correlated with SGK1 mRNA ( $r=$ $0.46, p=0.04$, and $r=0.63, p=0.005$, respectively).

Conclusions $\mathrm{Na}$-K-ATPase $\alpha 1$ is highest during early adaptation coinciding with the challenge of fluid absorption during immediate postnatal life. High SGK1 may be related perinatal stress. SGK1 dependent induction of $\mathrm{ENaC}$ and $\mathrm{Na}-\mathrm{K}$-ATPase may be an important physiological mechanism for lung fluid clearance.

\section{THE COMPARISON OF FORKHEAD BOX M1 MRNA EXPRESSION OF LUNG TISSUES BETWEEN PRETERM AND TERM RABBITS}

doi:10.1136/archdischild-2012-302724.1807

J Chang, CW Bae. Pediatrics, Kyung Hee University Hospital at Gangdong, Seoul, Republic of Korea

Background Recent reports on Forkhead box m1 (Foxm1) of the mice provided correlations between this gene and lung maturation. However, there has been no study on human Foxm1 concerned with lung maturation. The purposes of this study are to compare the mRNA expression of SP-A, $-\mathrm{B},-\mathrm{C}$ and Foxm1 gene of preterm rabbits to that of mature term ones and to trace the relationship between Foxm 1 and lung maturation.

Methods Pregnant New Zealand White rabbits were grouped according to gestational age. The cesarean sections were carried out after the group was divided into two groups of 30 31 days of gestation (Term group) and 26-27 days of gestation (Preterm group). The numbers of fetus rabbits of each group were 18. We compared the expression levels of mRNA of SP-A, -B, -C and Fxom1 by using RTPCR and real-time RT-PCR (qRT-PCR).

Results When relative ratio of SP-A, $-\mathrm{B}$, and -C mRNA expression level of term group was 1 , there were markedly decreased expressions of them in preterm group $-0.380,0.563$, and 0.448 respectively in order in qRT-PCR. On the contrary to these results, Foxm1 expression was increased in preterm group and its relative expression ratio was 1: 2.166 on both RT-PCR and real-time RT-PCR $(P<0.01)$.

Conclusion The preterm rabbits showed two times more mRNA expression of Foxm1 gene in their lungs than full terms. This Foxm 1 is the gene associated for lung maturation of preterm rabbits.

\section{PORACTANT ALFA THERAPY ASSOCIATED WITH C-REACTIVE PROTEIN RISE}

doi:10.1136/archdischild-2012-302724.1808

'MP Sherman, 'LR Breedlove, 1,2J Sherman. 'Child Health; ${ }^{2}$ Sinclair School of Nursing, University of Missouri - Columbia, Columbia, MO, USA

Background and Aims French and Finnish studies report a rise in C-reactive protein $[\mathrm{CRP}]$ after poractant alfa [PA] therapy; we have made a similar observation. Neither study excluded perinatal infection as a cause. This research hypothesized that the rise in CRP was not caused by infection but rather by a reaction to PA.

Methods This study reviewed newborns weighing $<1500 \mathrm{~g}$ at birth with respiratory distress syndrome [RDS] and who received PA. Clinical and radiographic criteria defined RDS. Clinical and laboratory findings established that infection was not present in the mother or infant (inclusion criteria). Infants given PA were compared to infants with RDS and no therapy [NO-PA]. A CRP measurement $\geq 1 \mathrm{mg} / \mathrm{dL}$ was considered elevated. SPSS was used for statistical analyses.

Results The $2^{\text {nd }}$ and $3^{\text {rd }}$ CRP rose in PA v. a decline in NO-PA [Table]. Tracheal aspirate and blood cultures had no growth in all subjects. 